\title{
Distal transradial access as default approach for coronary angiography and interventions
}

\author{
Marcos Danillo Peixoto Oliveira ${ }^{1}$, Ednelson Cunha Navarro ${ }^{1}$, Ferdinand Kiemeneij ${ }^{2 *}$ \\ ${ }^{1}$ Department of Interventional Cardiology, Hospital Regional do Vale do Paraíba, Taubaté, São Paulo, Brazil; ${ }^{2}$ Interventional Cardiologist, the \\ Netherlands \\ Contributions: (I) Conception and design: MDP Oliveira; (II) Administrative support: MDP Oliveira; (III) Provision of study materials or patients: \\ MDP Oliveira, EC Navarro; (IV) Collection and assembly of data: MDP Oliveira, EC Navarro; (V) Data analysis and interpretation: MDP Oliveira, \\ F Kiemeneij; (VI) Manuscript writing: All authors; (VII) Final approval of manuscript: All authors. \\ Correspondence to: Marcos Danillo Peixoto Oliveira. Department of Interventional Cardiology, Hospital Regional do Vale do Paraíba, Avenida \\ Tiradentes, 280, Jardim das Nações, Taubaté, 12030-180, São Paulo, Brazil. Email: mdmarcosdanillo@gmail.com.
}

Background: Distal transradial access (dTRA) as a refinement of the conventional transradial access has advantages in terms of patient and operator comfort and risk of radial artery (RA) occlusion. RA preservation with this new technique could be a relevant issue in patients requiring its future use. In turn, one relevant drawback is the more challenging puncture of a smaller artery. In order to evaluate the real world feasibility and safety of both right (rdTRA) and left (ldTRA) distal transradial access as default access site for routine coronary angiography (CAG) and percutaneous coronary intervention (PCI), this prospective observational registry was conducted.

Methods: From February to July 2019, 435 consecutive patients underwent CAG and/or PCI (620 procedures at all, by two experienced transradial operators) through rdTRA or ldTRA.

Results: Mean patient age was 62.4 years old. Most were male (66.0\%). The majority (49.4\%) of patients had an acute coronary syndrome; overall, 15.2\% with ST-elevation acute myocardial infarction (STEMI). Distal RA was successfully punctured in all patients, always without ultrasound guidance, with puncture and sheath insertion at until 2 attempts in the vast majority of patients. We had only $3.0 \%$ access site crossovers (successful arterial puncture but failed sheath insertion), mainly performed via the contralateral dTRA (53.8\%). Successful dTRA sheath insertion was then achieved in $98.6 \%$ of all 435 patients. Redo ipsilateral dTRA was performed in $2.5 \%$ of patients. Distal and proximal RA pulses were palpable in all patients at hospital discharge. No major adverse cardiac and cerebrovascular events and no major complications were recorded.

Conclusions: dTRA as default approach for routine CAG and/or PCI by experienced transradial operators appears to be safe and feasible. Further randomized and larger trials are still needed to assure the clinical benefits and the safety of this new technique.

Keywords: Distal transradial access (dTRA); coronary interventions

Submitted Aug 02, 2019. Accepted for publication Aug 30, 2019.

doi: $10.21037 / \mathrm{cdt} .2019 .09 .06$

View this article at: http://dx.doi.org/10.21037/cdt.2019.09.06

${ }^{*}$ Currently no affiliation. 


\section{Introduction}

Described for the first time by Babunashvili et al. (1) for retrograde recanalization of occluded ipsilateral radial arteries, the left distal transradial access (ldTRA) in the anatomical snuffbox (radial fossa) was recently described in detail as default approach by Kiemeneij (2).

As a refinement of the conventional proximal transradial access (pTRA), this new technique has advantages in terms of both patient and operator comfort and risk of proximal radial artery (RA) occlusion. RA preservation is imperative in patients requiring its future use for hemodialysis fistula preparation, coronary artery bypass grafting (CABG), and, most important, repeat TRA procedures (3). In turn, relevant drawbacks of the dTRA are the more challenging puncture of a smaller artery, with a steeper learning curve, and the higher rate of failure to advance the wire and then to cannulate the distal RA due to increased angulations at this point $(3,4)$.

In order to evaluate the real world feasibility and safety of both right and left dTRA as default access sites for routine coronary angiography (CAG) and percutaneous coronary intervention (PCI), this prospective observational registry was conducted.

\section{Methods}

Ethical approval from the hospital committee was obtained and informed consent was given as a prerequisite before enrolling each subject in this prospective registry. Patients were selected from the catheterization laboratory of Hospital Regional do Vale do Paraíba, a Brazilian tertiarycare hospital, between February and July 2019.

The presence of any (even weak) palpable pulse in both the wrist and the anatomical snuffbox was the only eligibility criterion for the enrolment.

Due to the increased confidence of the two operators, patients with unstable hemodynamic conditions were not excluded.

\section{Technical aspects of the dTRA procedure}

For ldTRA procedures, the patient's left upper arm was folded over his (her) belly toward the operator, who was always standing on the right side of the patient. For right distal transradial access (rdTRA), patient's right upper arm was placed on a side board with the hand in a neutral position. The choice of the site of dTRA (left or right) was on operating physician discretion and patient preference after detailed evaluation of the patient's pulses, medical history and clinical case characteristics.

The patient was asked to grasp his thumb under the other four fingers in order to bring the distal RA to the surface of the radial fossa, with the hand slightly abducted.

After subcutaneous injection of $2-3 \mathrm{~mL}$ lidocaine hydrochloride through a $25 \mathrm{G}$ needle, the distal RA was punctured proximal from the extensor pollicis longus tendon in the anatomical snuffbox using a $20 \mathrm{G}$ micropuncture cannula over needle with the Seldinger's technique, under an angle of 30-45 degrees, from lateral to medial, into the direction of the traditional (wrist) course of the RA. The through-and-through puncture (operator preference) was always performed with special caution, since the contact of the needle to the periostium of scaphoid and trapezium bones can be painful.

After successful arterial puncture, with brisk back flow, a flexible, soft, straight 0.021 ' hydrophilic guidewire was smoothly advanced through the cannula and then used as a rail to guide the sheath advancement through the artery. Almost all of our cases (97.6\%) were performed using a short $10 \mathrm{~cm}$ hydrophilic radial 6 Fr sheath Radifocus ${ }^{\circledR}$ Introducer II Standard Kit (Terumo Corp., Tokyo, Japan), since this one is the standard device in our cath lab.

After confirmation of the arterial waveform on the monitor followed by the intra-arterial administration of a spasmolytic cocktail (200 mcg of nitroglycerine plus saline) and a dose of heparin (5,000 IU), the operator was then able to take up the position at the level of the patient's knees to manipulate the 0.35 " wire, the catheters and the intracoronary devices. CAG and/or PCI were performed in the usual manner. The additional weight-adjusted dose of heparin was administered in case of PCI. The $5 \mathrm{Fr}$ diagnostic TIG ${ }^{\circledR}$ catheter (Terumo Corp., Tokyo, Japan) was used as the initial default option for all patients.

At the end of the procedure, the sheath was pulled out for $5 \mathrm{~cm}$, and a TR BAND ${ }^{\circledR}$ Radial Compression Device (Terumo Corp., Tokyo, Japan) haemostatic band was placed over the puncture site (Figure 1). Due to the fact that this device was not originally designed for this puncture site, adhesive tape reinforcement was routinely used to optimize its fixation. When the device was not available and in patients with a wrist circumference larger than that of the band, a haemostatic pad of wrapped gauze was placed over the puncture site using adhesive tape with a firm pressure for compression (Figure 1). The TR band (or the 

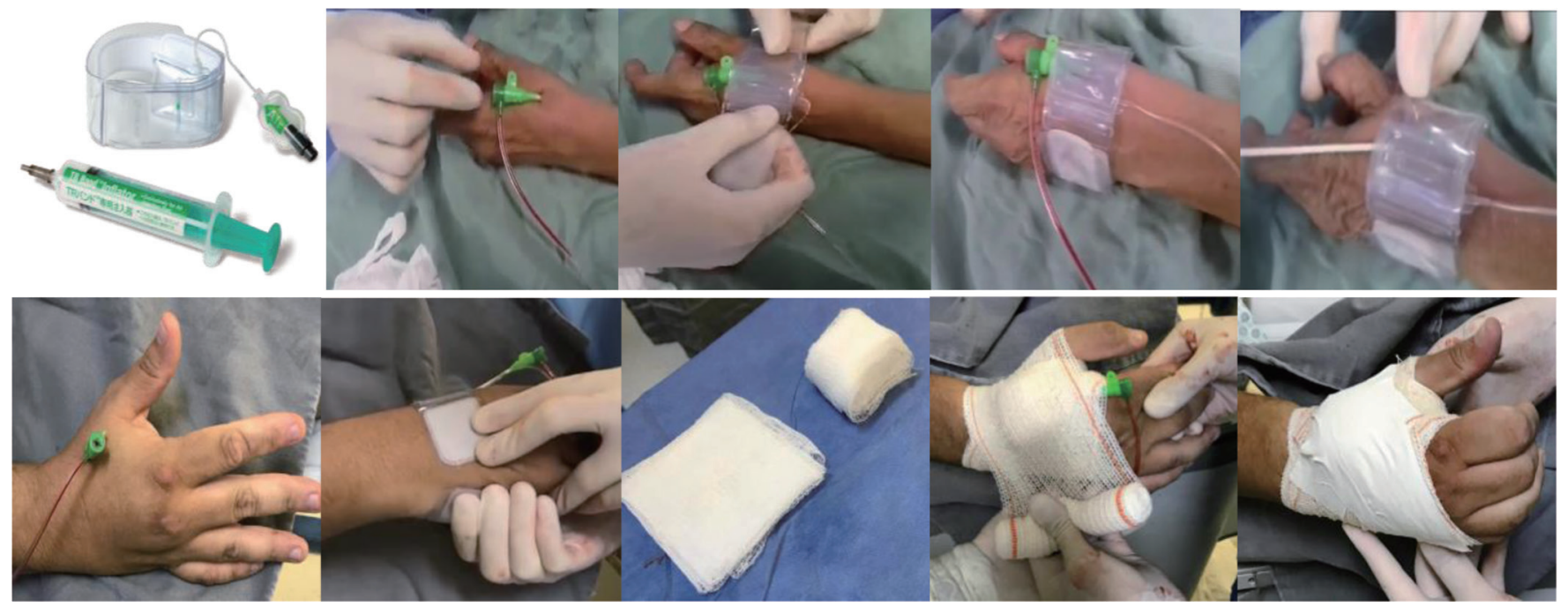

Figure 1 Superior panels: hemostasis with TR BAND ${ }^{\circledR}$ Radial Compression Device adaptation for dTRA. Inferior panels: haemostatic pad of wrapped gauze and adhesive tape in a patient with wrist circumference larger than that of the band.

gauze compressive bandage) was left in situ and completely removed within two hours in all patients. Before hospital discharge, the presence of a radial pulse at the distal forearm and in the anatomical snuffbox was carefully checked by manual palpation in all patients.

\section{Statistical analysis}

All analyses were performed with the Statistical Package for Social Science, version 24 (IBM). Continuous variables are reported as mean \pm standard deviation. Categorical data are reported as numbers and percentages.

\section{Results}

Table 1 summarizes the baseline characteristics of all 435 patients. Mean patient age was 62.4 years old. Most were male $(66.0 \%)$. The majority of patients enrolled had an acute coronary syndrome (UA, 9.9\%; NSTEMI, 23.4\%; STEMI 15.2\%) and was then submitted to urgency CAG.

Table 2 presents the total 620 procedural characteristics. Successful dTRA sheath insertion was achieved in $429(98.6 \%)$ patients, with puncture and sheath insertion at until 2 attempts in the vast majority of patients. Of note, among the total 435 patients, we had only $13(3.0 \%)$ access site crossovers. The distal RA was successfully punctured (with a brisk flow through the catheter) in all patients, always without ultrasound guidance, but wire insertion and subsequent arterial cannulation were not possible, in these cases, mainly due to distal and/or forearm RA hypoplasia, tortuosities, angulations and/or loopings. The most usual crossover was from initial (failure) ldTRA to rdTRA (7 in 13 cases, $53.8 \%)$.

Primary and rescue PCI were performed in 53 (8.5\%) and $2(0.3 \%)$ patients, respectively. The LAD (and its diagonal branches) was the most common coronary artery territory treated by PCI, followed by the RCA and the LCx.

TR BAND ${ }^{\circledR}$ Radial Compression Device (used in $94.9 \%$ of patients) or the gauze compressive bandage were left in situ and completely removed within 2 hours in all patients, according to institutional protocols.

On average, the amount of contrast used was $79.6 \mathrm{~mL}$. Mean fluoroscopy and procedural (from first puncture attempt to sheath removal) times were $4.38 \mathrm{~min}$ and $16.5 \mathrm{~min}$, respectively. The "fluor store" resource (recording up to the last 30 seconds of fluoroscopy) was routinely used by the operator in order to reduce both contrast volume and radiation exposure.

There were eleven cases of redo ipsilateral dTRA. All of those procedures were performed without any difficulties or complications. Two cases of unknown chronic total occlusion of the left subclavian artery were incidentally discovered after the initial successful ldTRA for elective CAG and PCI, respectively. Since it was not possible to reach the ascending aorta, the ITRA was used for the proposed procedures (bilateral dTRA, Figure 2). In one 
Table 1 Baseline characteristics of all 435 patients

\begin{tabular}{|c|c|}
\hline Patient characteristics (total $n=435$ patients) & Values \\
\hline Age, years & $62.4 \pm 9.5$ \\
\hline Height (cm) & $178.5 \pm 3.5$ \\
\hline Weight (kg) & $87.0 \pm 9.0$ \\
\hline Men & $287(66.0 \%)$ \\
\hline Hypertension & $353(81.1 \%)$ \\
\hline Diabetes mellitus & $169(38.9 \%)$ \\
\hline Current smoking & $85(19.5 \%)$ \\
\hline Former smoking & $130(29.9 \%)$ \\
\hline Obesity & $52(12.0 \%)$ \\
\hline Known coronary artery disease & $122(28.0 \%)$ \\
\hline Previous acute myocardial infarction & $60(13.8 \%)$ \\
\hline Previous percutaneous coronary intervention & $66(15.2 \%)$ \\
\hline Previous coronary artery bypass grafting & $28(6.4 \%)$ \\
\hline Previous ipsilateral pTRA sheath insertion & $13(3.0 \%)$ \\
\hline Redo ipsilateral dTRA & $11(2.5 \%)$ \\
\hline $\begin{array}{l}\text { Chronic kidney disease without dialysis } \\
(\text { eGFR }<60)\end{array}$ & $27(6.2 \%)$ \\
\hline Chronic kidney disease under dialysis & $10(2.3 \%)$ \\
\hline \multicolumn{2}{|l|}{ Indication for coronary angiography } \\
\hline Stable angina pectoris & $196(45.1 \%)$ \\
\hline Unstable angina & $43(9.9 \%)$ \\
\hline NSTEMI & $102(23.4 \%)$ \\
\hline STEMI & $66(15.2 \%)$ \\
\hline Anterior STEMI & $32(48.5 \%)$ \\
\hline Inferior STEMI & $24(36.4 \%)$ \\
\hline Infero-lateral STEMI & $8(12.1 \%)$ \\
\hline Lateral STEMI & $2(3.0 \%)$ \\
\hline Cardiogenic shock & $4(0.9 \%)$ \\
\hline Severe aortic stenosis & $10(2.3 \%)$ \\
\hline Other reasons & $14(3.2 \%)$ \\
\hline
\end{tabular}

Data presented as mean \pm standard deviation or number (percentage). pTRA, proximal transradial access; dTRA, distal transradial access; eGFR, estimated glomerular filtration rate; NSTEMI, non-ST-elevation myocardial infarction; STEMI, STelevation myocardial infarction.
Table 2 Baseline characteristics of all 620 procedures in 435 patients

\begin{tabular}{|c|c|}
\hline $\begin{array}{l}\text { Procedural characteristics } \\
\text { (620 procedures in } 435 \text { patients) }\end{array}$ & Values \\
\hline Elective coronary angiography & $173(27.9 \%)$ \\
\hline Urgent coronary angiography & $217(35.0 \%)$ \\
\hline Elective percutaneous coronary intervention & $44(7.1 \%)$ \\
\hline $\begin{array}{l}\text { Primary percutaneous coronary intervention } \\
\text { (following urgent CAG) }\end{array}$ & $53(8.5 \%)$ \\
\hline $\begin{array}{l}\text { Rescue percutaneous coronary intervention } \\
\text { (following urgent CAG) }\end{array}$ & $2(0.3 \%)$ \\
\hline $\begin{array}{l}\text { Ad hoc percutaneous coronary intervention } \\
\text { (following urgent or elective CAG) }\end{array}$ & $130(21.0 \%)$ \\
\hline Elective cerebral angiography & $1(0.2 \%)$ \\
\hline Coronary artery territory treated by $\mathrm{PCI}$ & 226 \\
\hline Left main & $3(1.3 \%)$ \\
\hline $\begin{array}{l}\text { Left anterior descending artery and/or } \\
\text { diagonal branches }\end{array}$ & $97(42.9 \%)$ \\
\hline $\begin{array}{l}\text { Left circumflex artery and/or obtuse } \\
\text { marginal branches }\end{array}$ & $55(24.3 \%)$ \\
\hline Right coronary artery and/or branches & $70(31 \%)$ \\
\hline SVG-RCA & $2(0.8 \%)$ \\
\hline SVG-LAD & $1(0.4 \%)$ \\
\hline SVG-LCx & $1(0.4 \%)$ \\
\hline LIMA-LAD & $1(0.4 \%)$ \\
\hline $\begin{array}{l}\text { Successful dTRA sheath insertion } \\
\text { (total: } 435 \text { patients) }\end{array}$ & $429(98.6 \%)$ \\
\hline IdTRA & $153(35.2 \%)$ \\
\hline redo IdTRA & $2(0.5 \%)$ \\
\hline rdTRA & $255(58.6 \%)$ \\
\hline redo rdTRA & $9(2.1 \%)$ \\
\hline rdTRA after failed initial IdTRA & $7(1.6 \%)$ \\
\hline Bilateral dTRA (IdTRA and rdTRA) & $3(0.7 \%)$ \\
\hline Sheath size (5 Fr) & $5(0.8 \%)$ \\
\hline Sheath size (6 Fr) & $605(97.6 \%)$ \\
\hline Sheath size $(7 \mathrm{Fr})$ & $4(0.6 \%)$ \\
\hline \multicolumn{2}{|l|}{ Hemostasis of dTRA (435 patients) } \\
\hline TR BAND radial compression device $®$ & $413(94.9 \%)$ \\
\hline Gauze compressive bandage & $18(4.1 \%)$ \\
\hline
\end{tabular}

Table 2 (continued) 
Table 2 (continued)

\begin{tabular}{|c|c|}
\hline $\begin{array}{l}\text { Procedural characteristics } \\
\text { (620 procedures in } 435 \text { patients) }\end{array}$ & $N(\%)$ \\
\hline $\begin{array}{l}\text { Crossover to another access site ( } 435 \\
\text { patients) }\end{array}$ & $13(3.0 \%)$ \\
\hline IdTRA failure $\rightarrow$ right pTRA successful & $1(7.7 \%)$ \\
\hline $\begin{array}{l}\text { IdTRA failure } \rightarrow \text { left pTRA (LIMA-LAD) } \\
\text { successful }\end{array}$ & $1(7.7 \%)$ \\
\hline $\begin{array}{l}\text { IdTRA failure } \rightarrow \text { left pTRA (LIMA-LAD) } \\
\text { failure } \rightarrow \text { right TFA successful }\end{array}$ & $1(7.7 \%)$ \\
\hline IdTRA failure $\rightarrow$ rdTRA successful & $7(53.8 \%)$ \\
\hline rdTRA failure $\rightarrow$ right pTRA successful & $3(23.1 \%)$ \\
\hline Procedural time (min) & $16.5 \pm 13.9$ \\
\hline Fluoroscopy time (min) & $4.38 \pm 4.46$ \\
\hline Cumulative air kerma (mGy) & $1,141.4 \pm 1,020.8$ \\
\hline $\operatorname{DAP}\left(\mathrm{mGy} \cdot \mathrm{cm}^{2}\right)$ & $6,044.8 \pm 8,687.9$ \\
\hline Contrast volume (mL) & $79.6 \pm 40.0$ \\
\hline
\end{tabular}

Data presented as mean \pm standard deviation or number (percentage). CAG, coronary angiography; $\mathrm{PCI}$, percutaneous coronary intervention; SVG-RCA, saphenous vein graft-right coronary artery; LIMA-LAD, left internal mammary artery-left anterior descending; dTRA, distal transradial access; IdTRA, left distal transradial access; rdTRA, right distal transradial access; Fr, French; pTRA, proximal transradial access; TFA, transfemoral access; DAP, dose-area product; RA, radial artery.

patient, diagnostic cerebral angiography was successfully performed via rdTRA.

Ten $(2.2 \%)$ out of the 435 patients were already under hemodialysis via upper arm arteriovenous fistulae and 27 $(6.2 \%)$ were possible future candidates to it due to advanced stages of CKD. In those patients, the pTRA has historically being avoided in order to preserve the RA.

Neither major adverse cardiac and cerebrovascular events nor major ischemic or bleeding complications were recorded. No patient complained of local numbness or dysfunction of the hand after the procedure. Distal and proximal RA pulses were palpable in all patients at hospital discharge.

\section{Discussion}

This is the first Brazilian prospective observational registry designed to evaluate dTRA as default approach for performing routine CAG and/or PCI. We demonstrated that the high success and no major complication rates support the feasibility and safety of this new technique.

In this our initial experience with dTRA, the distal RA was successfully punctured in all patients and we had only $3.0 \%$ (13 out of 435 patients) access site crossovers, which were mainly performed via the contralateral dTRA (7 out of 13 patients, $53.8 \%$ ). Successful dTRA sheath insertion was then achieved in $98.6 \%(429 / 435)$ of the patients: $59.4 \%$ rdTRA, 35.6\% ldTRA, 2.0\% redo rdTRA, 1.6\% rdTRA after failed initial ldTRA, $0.7 \%$ bilateral dTRA and $0.4 \%$ redo ldTRA.

Distal and proximal RA pulses were palpable in all patients at hospital discharge. However, this method is not reliable for excluding RA occlusion due to the presence of a strong collateral network. Despite the many advantages of the ultrasound evaluation, like proper assessment of RA diameters and establishment of postprocedural patency, this resource was not available in our cath lab.

In the first series reported by Kiemeneij, 70 patients underwent ldTRA. Puncture was not attempted in case of weak or absent pulse, logistical reasons, indwelling venous cannula, left-handedness, and patient preference. Eight patients (11\%) had a failed ldTRA: in half puncture failed, while in the other half puncture was successful, but wire could not be advanced towards the forearm part of the RA. As in our current registry, no major bleeding nor thumb or hand dysfunction were encountered. At followup assessment, all RA were open at the conventional forearm site and one patient had an occluded distal RA (2). Of note, as a pioneer, Kiemeneij had no information on how to perform the procedure, like videos, manuscripts or presentations, so the high failure rate might be explained.

In the LeDRA Korean prospective observational registry, 200 patients were enrolled in a single center, by three operators, after a 3 months (30 cases) period of adaptation with the ldTRA technique (unlike in our study, in which all patients were included without such a period of initial adaptation). The success rates of arterial puncture, CAG, and PCI were $95.5 \%, 100 \%$ and $98.9 \%$, respectively. There were $7.4 \%$ puncture site minor hematomas and $0.5 \%$ arterial dissection. Two patients complained of left thumb numbness at one-month follow-up. No distal RA occlusion, perforation, pseudoaneurysm, or arteriovenous fistula occurred (5).

Valsecchi et al reported $90 \%$ success in a series of 52 patients undergoing right or left dTRA (79\% rdTRA). Considering failure due to RA occlusion, not discernible by radial pulse, the overall success rate rose to $94 \%$ 

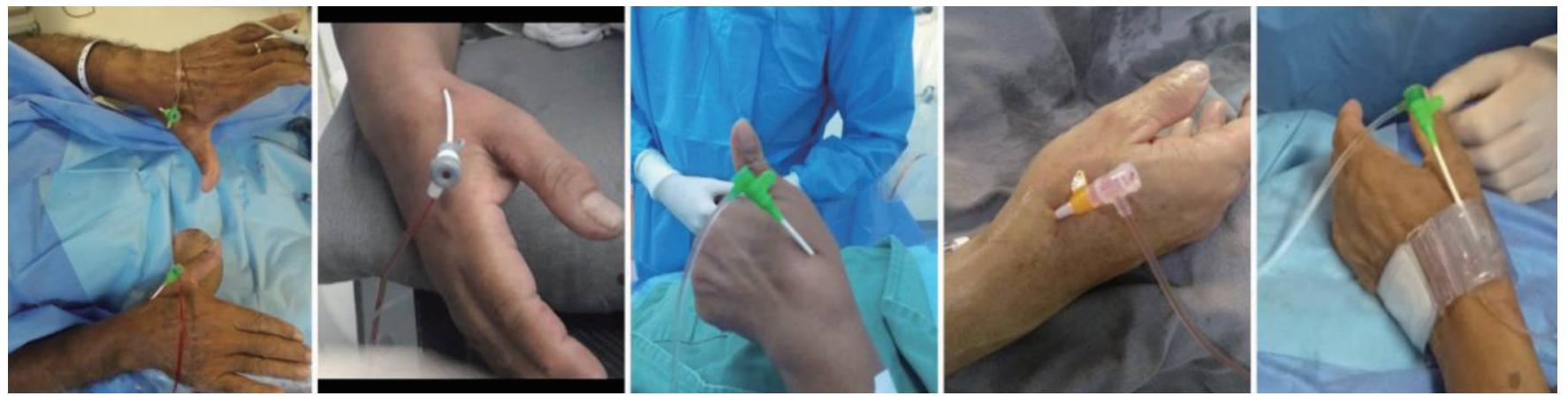

Figure 2 Different sheath sizes (5, 6 and 7 Fr) in place (bilateral dTRA, rdTRA and ldTRA), at the end of the procedures.

(47/50 patients). No complications were reported (6). Ziakas et al., in a two-center study utilizing exclusively the rdTRA in 49 consecutive patients, reported a $10.2 \%$ failed attempt. No distal or forearm RA occlusion was observed on triplex ultrasonography $24 \mathrm{~h}$ after successful hemostasis. No major complications were recorded (7).

Andrade et al., in a Brazilian multicenter initial experience with the ldTRA in 61 patients, had a $4.9 \%$ rate of cannulation failure, with no major vascular complications. In one patient, mild ecchymosis was observed, with concomitant asymptomatic distal RA occlusion (8). We report herein the initial results from the first Brazilian prospective single center dTRA registry, with seven fold the number of patients with similar demographic characteristics.

In the first randomized comparison of distal versus traditional radial approach for CAG in 200 patients, Koutouzis et al. described a surprisingly $30 \%$ rate (10 folds higher than ours) of access site crossover (the primary endpoint of the trial) in the dTRA group in contrast to only $2 \%$ in the traditional arm $(\mathrm{P}<0.001)(4)$.

We decided to include patients with any (even weak) right and/or left distal RA palpable pulse, regardless the clinical scenario. Of note, the majority $(49.4 \%)$ of patients enrolled had an acute coronary syndrome and four were in cardiogenic shock (all successful rdTRA at first attempt).

Particularly for ldTRA, in comparison to the classic left pTRA, since the left upper arm can be positioned over the patient's belly towards the right inguinal region and the sheath is inserted on the dorsal aspect of the hand, catheters can be more easily handled without the need to lean over the patient, with greater patient and operator comfort. For dTRA, once the arterial puncture is performed after the point of emergence of the superficial palmar branch, in case of RA occlusion, blood flow through the palmar arch would not be compromised and the risk of ischemic injury would be minimal. Also, by avoiding the conventional puncture site and subsequent related complications seen on imaging studies, the RA would be preserved for future uses $(3,8)$.

The short length of the standard radial catheters may be an important drawback of the dTRA. Given that distal RA is up to $5 \mathrm{~cm}$ below the pTRA entry site, these catheters may therefore be too short, especially in taller patients, and operators may have to perform CAG and/or PCI "on the tip" of the catheter.

If it is proven in larger populations that dTRA almost eliminates RA occlusion, this could be one of the major advantages of this new technique compared to the pTRA. Increasing personal experience will result in improvements in the learning curve, and gathered center experience will delineate further aspects of the technique.

On the basis of shared results, personal experience and quality control, dTRA has become, since the beginning of this registry, the operators' default approach for CAG and PCI.

\section{Study limitations}

This is a single center registry, and all procedures were performed by two interventional cardiologists with vast experience in transradial access. The absence of a control group limits our assumptions. Despite the presence of proximal and distal RA pulse by manual palpation in all patients at discharge, the lack of post-procedural routine Doppler ultrasonography evaluation could have led to an underestimation of access site vascular complications.

\section{Conclusions}

dTRA as default approach for routine CAG and PCI by experienced transradial operators appears to be safe and 
feasible. Further randomized and larger trials are still needed in order to assure the clinical benefits and the safety of this new technique.

\section{Acknowledgments}

To all the members of the nursing staff of our cath lab, for their indispensable commitment with the adoption of dTRA as the new default approach. To Dr. Glenda Alves de Sá, for her special support during the course of this study.

\section{Footnote}

Conflicts of Interest: The authors have no conflicts of interest to declare.

Ethical Statement: The authors are accountable for all aspects of the work in ensuring that questions related to the accuracy or integrity of any part of the work are appropriately investigated and resolved. Ethical approval from the hospital committee was obtained and informed consent was given as a prerequisite before enrolling each subject in this prospective registry.

\section{References}

1. Babunashvili A, Dundua D. Recanalization and reuse of early occluded radial artery within 6 days after previous

Cite this article as: Oliveira MDP, Navarro EC, Kiemeneij F. Distal transradial access as default approach for coronary angiography and interventions. Cardiovasc Diagn Ther 2019;9(5):513-519. doi: 10.21037/cdt.2019.09.06 transradial diagnostic procedure. Catheter Cardiovasc Interv 2011;77:530-6.

2. Kiemeneij F. Left distal transradial access in the anatomical snuffbox for coronary angiography (ldTRA) and interventions (ldTRI). EuroIntervention. 2017;13:851-7.

3. Sgueglia GA, Di Giorgio A, Gaspardone A, et al. Anatomic Basis and Physiological Rationale of Distal Radial Artery Access for Percutaneous Coronary and Endovascular Procedures. JACC Cardiovasc Interv 2018;11:2113-9.

4. Koutouzis M, Kontopodis E, Tassopoulos A, et al. Distal Versus Traditional Radial Approach for Coronary Angiography. Cardiovasc Revasc Med 2019;20:678-80.

5. Lee JW, Park SW, Son JW, et al. Real-world experience of the left distal transradial approach for coronary angiography and percutaneous coronary intervention: a prospective observational study (LeDRA). EuroIntervention 2018;14:e995-1003.

6. Valsecchi O, Vassileva A, Cereda AF, et al. Early Clinical Experience With Right and Left Distal Transradial Access in the Anatomical Snuffbox in 52 Consecutive Patients. J Invasive Cardiol 2018;30:218-23.

7. Ziakas A, Koutouzis M, Didagelos M, et al. Right arm distal transradial (snuffbox) access for coronary catheterization: Initial experience. Hellenic J Cardiol 2018. [Epub ahead of print].

8. Andrade PB, Tebet MA, Rinaldi FS, et al. Initial experience with left distal transradial access for invasive coronary procedures. J Transcat Intervent 2018;26:eA0002. 